\title{
Application of NEAT for determining the composition dependence of activity coefficients in poorly specified mixtures
}

\author{
Fabian Jirasek ${ }^{\mathrm{a}, *}$, Jakob Burger ${ }^{\mathrm{b}}$, Hans Hasse $^{\mathrm{a}}$ \\ ${ }^{a}$ Laboratory of Engineering Thermodynamics (LTD), Technische Universität Kaiserslautern, \\ Erwin-Schrödinger-Str. 44, 67663 Kaiserslautern, Germany \\ ${ }^{b}$ Chair of Chemical Process Engineering, Technical University of Munich, Campus Straubing for Biotechnology \\ and Sustainability, Schulgasse 16, 94315 Straubing, Germany
}

\begin{abstract}
Poorly specified mixtures, of which the composition is only partially known, are important in many fields. In a recent work of our group, the method NEAT for estimating activity coefficients of target components in such mixtures was introduced. In the present work, it is shown that based on a single NMR analysis of a poorly specified mixture not only the activity coefficient of the target component in that mixture but also its composition dependence can be predicted with NEAT. Hence, based on a single NMR analysis, the activity coefficient of the target component in many mixtures can be predicted with NEAT. This is possible, if the unknown part of the mixture remains unchanged, which is the case e.g. when the target component is selectively removed or when a known solvent is added or removed. The approach is successfully tested using several aqueous test mixtures.
\end{abstract}

Keywords: NEAT, NMR spectroscopy, activity coefficients, unknown composition, varying composition

\section{Introduction}

Mixtures that contain components of unknown type or in an unknown concentration are called poorly specified mixtures here. They are common in many fields of engineering and science, such as petrochemical engineering [1 4, waste water treatment [5, 6], and biotechnology [7 9]. In process 5 design, such mixtures are hard to handle since classical thermodynamic methods, such as NRTL [10], UNIQUAC [11, 12], UNIFAC [13,15], or COSMO-RS [16, 17], require a full specification of the mixture. In a recent work of our group, the NEAT method (NMR Spectroscopy for the Estimation of Activity Coefficients of Target Components in Poorly Specified Mixtures) was introduced, which tackles this challenge [18, 19]. NEAT is based on a combination of NMR spectroscopy and thermodynamic group contribution methods and enables the estimation of the activity coefficients of known target components in poorly specified mixtures. In NEAT, the components of a poorly

\footnotetext{
*Corresponding author

Email address: fabian.jirasek@mv.uni-kl.de (Fabian Jirasek)
} 
specified mixture are divided in two classes: known and unknown components. For the known components, it is assumed that the mass fraction of the component in the mixture and its nature are known or can be obtained by established analytical methods. For the unknown components, no information is needed. NMR spectroscopy is used to estimate the type and the concentration of chemical groups of the unknown components in the mixture. This information on the groups is much easier to obtain than the corresponding information regarding the components. These data are then used for calculating the activity coefficients of the target components with a thermodynamic group contribution method. The target components can be selected arbitrarily among the known components. In our previous work [18, 19, modified UNIFAC (Dortmund) 15] was used as group contribution method. It was shown that based on a single ${ }^{1} \mathrm{H}$ or ${ }^{13} \mathrm{C}$ NMR spectrum of the poorly specified mixture, NEAT gives an excellent prediction for the activity coefficient of the target component: in tests in which the full composition of the mixtures was known but not used in NEAT, the results from NEAT agreed very well with those obtained from modified UNIFAC (Dortmund) for the fully specified mixtures. However, in those studies, one NMR spectrum of each considered mixture had to be taken.

In the following, the term 'system' is used to summarize all mixtures that contain the same components in different compositions. In process engineering, different mixtures of the same system occur frequently, e.g. during downstream processing due to the separation of target components or the solvent from a mixture or as result of a varying turnover in a reaction process. An individual NMR analysis of each mixture for the application of NEAT in these cases would be tedious.

In the present work, we study if based on the NMR analysis of a single poorly specified mixture, the activity coefficient of the target component not only in that mixture but also in other mixtures of the same system can be predicted with NEAT. Hence, we present an approach to determine the composition dependence of activity coefficients of target components in poorly specified mixtures based on a single NMR analysis, which makes the application of NEAT significantly more practical in many situations.

The approach is tested using several systems of the type (known target component + known solvent water + unknown components). The data on the studied systems are taken from our previous 40 work [18 and no experiments were carried out in the present work. In contrast to our previous work, in which the activity coefficient of the target component in several mixtures of a system was predicted based on a separate NMR analysis of each mixture, we use only one single NMR spectrum of a single mixture per system to predict the activity coefficients at a wide range of compositions here. The only condition is thereby that the composition of the unknown part of the mixtures, i.e. 45 the type and the ratio between the unknown components, remains constant. The predictions are compared to results obtained in our previous work for which an NMR spectrum of each studied 
mixture was used and to results that were calculated with modified UNIFAC (Dortmund) using the full knowledge on the composition of the mixtures.

\section{Application of NEAT}

A comprehensive description of the NEAT method has been given in Ref. 19]. The method itself is therefore not discussed in detail here.

All mixtures that are considered in the present work are of the type $\left(\mathrm{T}+\mathrm{W}+\mathrm{U}_{1} \ldots \mathrm{U}_{N}\right)$. Here, $\mathrm{T}$ is the target component of which the nature and the concentration in the mixture are known and $\mathrm{W}$ is a second component of which the nature is known. In all examples that are discussed here, that second component is the solvent water. The concentration of $\mathrm{W}$ in the mixture does not have to be known as it can be obtained from NEAT using a mass balance [19]. For the other components $\mathrm{U}_{1} \ldots \mathrm{U}_{N}$, nothing is known - neither their nature nor their concentration, and in general not even the number $N$ is known. These components are called unknown components. In NEAT, they are simply lumped together to a single mean unknown component $\tilde{\mathrm{U}}$, which is in contrast to using modified UNIFAC (Dortmund) or other group contribution methods, in which each component is considered explicitly. The nature and concentration of $\tilde{U}$ in the mixture is determined within NEAT from an NMR analysis as described in detail in Ref. [19].

After lumping the unknown components to $\tilde{\mathrm{U}}$, the studied mixture can be considered as pseudoternary mixture of the type $(T+W+\tilde{U})$. To calculate the activity coefficient of $T$ in NEAT, an activity model based on modified UNIFAC (Dortmund) is used here. However, also other activity models can be used for this purpose. This model is completely parametrized during the application of NEAT, cf. Ref. [19]. Thus, it is available for extrapolations to other mixtures of the same pseudo-ternary system $(T+W+\tilde{U})$ and for a given poorly specified mixture, variations of the concentrations of $\mathrm{T}, \mathrm{W}$, and $\tilde{\mathrm{U}}$ can be studied with NEAT. The only condition is thereby that the pseudo component $\tilde{U}$ does not change its nature, i.e. that the ratios of the amounts of $U_{i}$ $(i=1 \ldots N)$ are constant. There are many practical cases in which this is a good assumption, e.g. the addition or the selective removal of the target component $\mathrm{T}$ or the solvent $\mathrm{W}$.

If this assumption holds, only one single NMR spectrum of a single mixture has to be taken and the activity coefficients of $\mathrm{T}$ in all mixtures of the system $(\mathrm{T}+\mathrm{W}+\tilde{\mathrm{U}})$ can be predicted with 75 NEAT. The mixture of which the NMR spectrum is taken to obtain the information on $\tilde{U}$ is called reference mixture $\mathrm{R}$ here. The results of NEAT for the stoichiometry of $\tilde{\mathrm{U}}$ and the composition of the studied reference mixtures are summarized in Tables C.1-C.3 in the Appendix. There are no restrictions of the present method regarding the choice of the reference mixture R. If doubts arise, whether the basic assumption of the present work holds in a practical application, i.e. if there is reason to believe that the ratios of the $\mathrm{U}_{i}$ might vary, the following can be done: the assumption 
can be tested using two or more reference mixtures and applying NEAT to each of them. If the group speciation of $\tilde{U}$ is constant in the set, the assumption is good. If not, the procedure described in Refs. [18, 19] can be applied.

\section{Overview of the Present Study}

Table 1 gives an overview of the systems that were investigated in the present work. The experimental data on the reference mixtures were taken from our previous work [18]. In each system, two reference mixtures $R_{1}$ and $R_{2}$ were used. In principle, only one reference mixture is needed. Two of them were used here only to test whether the choice of the reference mixture has an influence on the results.

Quantitative ${ }^{13} \mathrm{C}$ NMR spectra of the reference mixtures were used to obtain information on the

Table 1: Overview of the studied systems and reference mixtures. Two reference mixtures $R_{1}$ and $R_{2}$ were used for comparisons in each system. Besides the target component $(\mathrm{T})$ and the unknown components $\left(\mathrm{U}_{i}\right)$, water $(\mathrm{W})$ was always present in the mixtures. No information on the components $\mathrm{U}_{i}$ was used in NEAT.

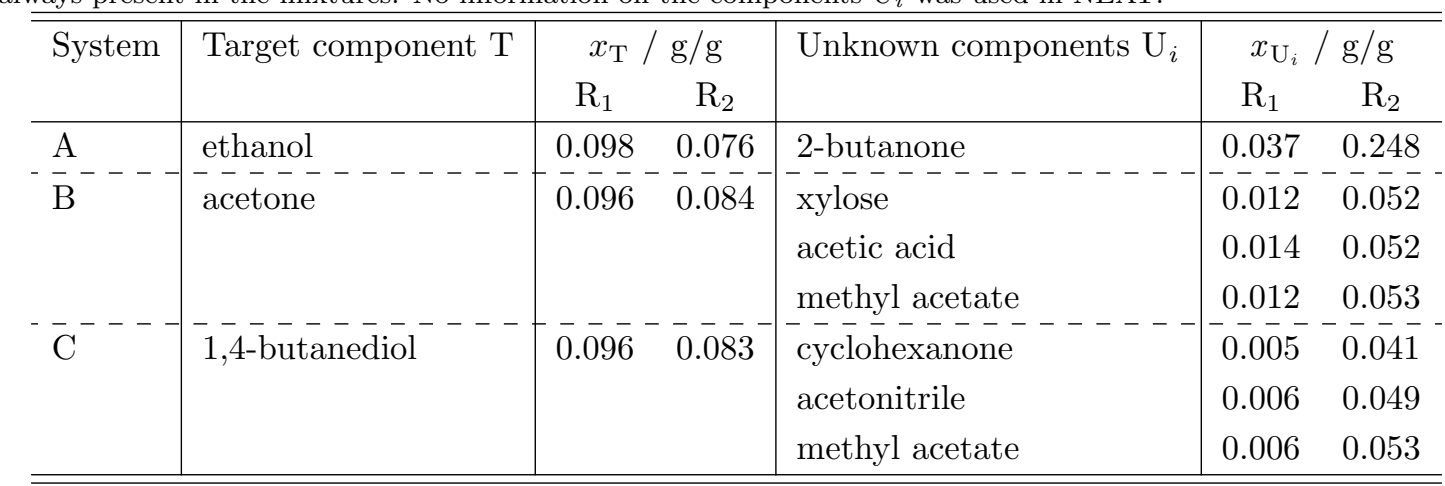

90

chemical groups of the unknown components in the studied systems. The assignment of chemical groups to chemical shift regions in the NMR spectra that was used in the present work is the same as the one that was used in our previous work [19] for aqueous mixtures and is given in Table A.1 the Appendix. The identified chemical groups of the unknown components were lumped to a mean unknown component $\tilde{\mathrm{U}}$, to which a molar mass of $M_{\tilde{\mathrm{U}}}=150 \mathrm{~g} / \mathrm{mol}$ was assigned as suggested in our previous work [19], in which was demonstrated that the value of $M_{\tilde{\mathrm{U}}}$ has only a minor influence on the predictions with NEAT, if not unreasonably small values are chosen. In principle, any mixture of the system can be chosen as reference mixture. The mass fraction of the target component $\mathrm{T}$ in that reference mixture was assumed to be known, that of the mean unknown component $\tilde{U}$ was obtained from NMR data of the mixture, and that of water was obtained using a mass balance [19]. Hence, for each poorly specified reference mixture R, the composition in the pseudo-ternary system $(\mathrm{T}+\mathrm{W}+\tilde{\mathrm{U}})$ was obtained from NEAT. Further, a modified UNIFAC (Dortmund) model to calculate activity coefficients in the pseudo-ternary system is provided by NEAT. This model is used to calculate the activity coefficient of the target component not only in 
the reference mixture $R$ but at any composition in the pseudo-ternary system $(T+W+\tilde{U})$. This is exemplarily shown in Figure 1 for system A.

In Figure 1, the symbol indicates the result for the activity coefficient of the target component

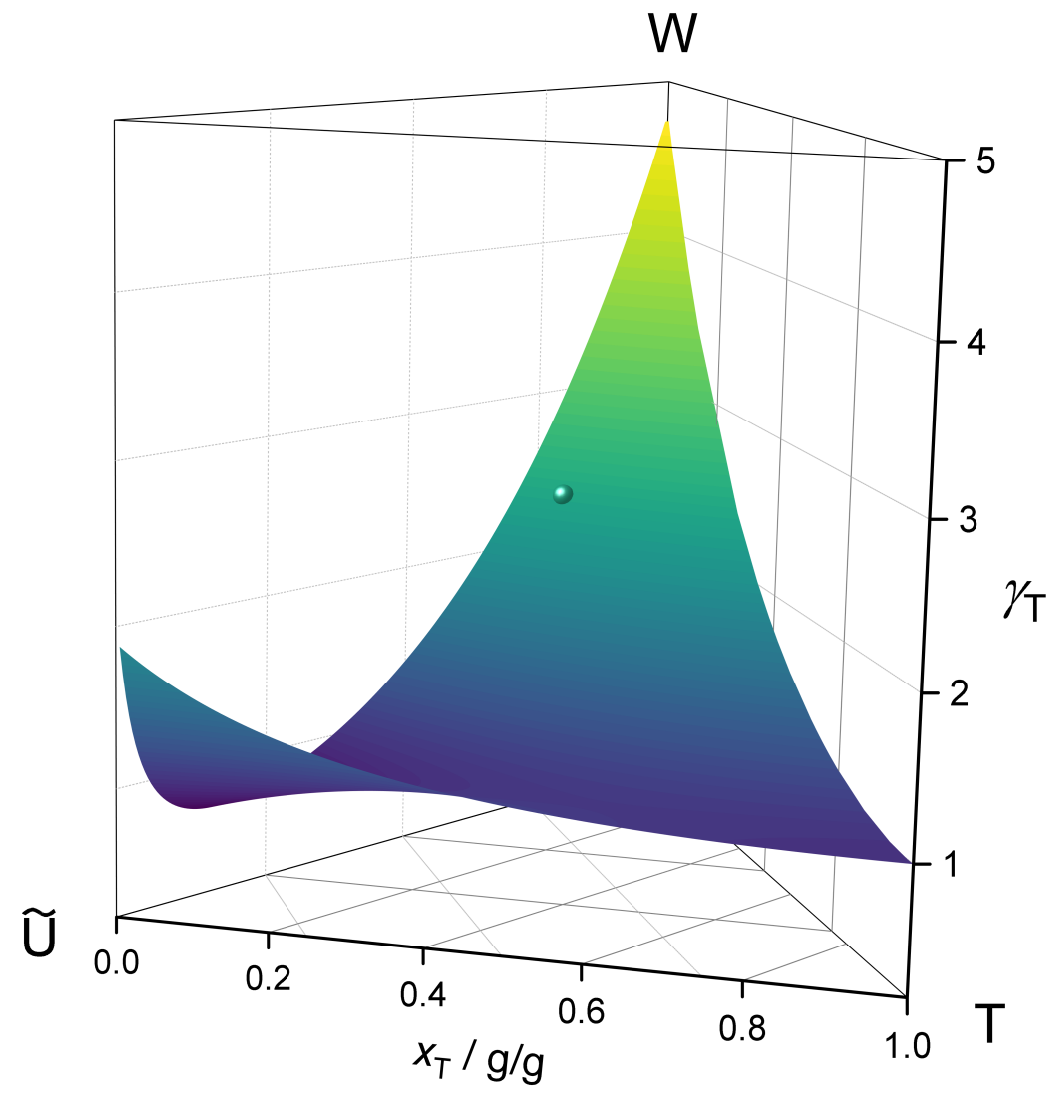

Figure 1: Prediction of the activity coefficient $\gamma_{\mathrm{T}}$ of target component ethanol $(\mathrm{T})$ in mixtures of system A based on the NMR analysis of reference mixture $\mathrm{R}_{2}$. The symbol indicates the result for $\mathrm{R}_{2}$. No information on $\mathrm{U}_{i}$ was used in NEAT. $T=298 \mathrm{~K}$.

( $\mathrm{T}=$ ethanol) in reference mixture $\mathrm{R}_{2}$ of system $\mathrm{A}$ as predicted with NEAT based on the NMR analysis of $R_{2}$. The surface indicates the activity coefficient of $T$ in all possible mixtures of the pseudo-ternary system $(\mathrm{T}+\mathrm{W}+\tilde{\mathrm{U}})$ as predicted with NEAT based on the same NMR analysis of $\mathrm{R}_{2}$.

Figure 1 shows only the predictions with NEAT for the pseudo-ternary system $(\mathrm{T}+\mathrm{W}+\tilde{\mathrm{U}})$. The results for each fully specified system $\left(\mathrm{T}+\mathrm{W}+\mathrm{U}_{1} \ldots \mathrm{U}_{N}\right)$ could in principle be shown in the same type of diagram, which requires summing the components $\mathrm{U}_{i}$ to $\tilde{\mathrm{U}}$ if $i>1$. For all studied systems, the respective surface calculated for the fully specified system was found to be very close to the surface predicted with NEAT. However, these results are not included in Figure 1 for improved illustration but shown in Figure B.1 in the Appendix together with a diagram representing the deviation between the results for the fully specified mixtures and the predictions with NEAT. For improved illustration of the accuracy of the predictions with NEAT, only the following variations were considered and the respective results are shown in the diagrams of the present work: 
starting from each reference mixture, the composition in the respective system of was varied in three ways as shown in Figure 2 in a schematic triangular diagram of the pseudo-ternary system $(\mathrm{T}+\mathrm{W}+\tilde{\mathrm{U}})$. The black squares denote the compositions of two reference mixtures $\mathrm{R}_{1}$ and $\mathrm{R}_{2}$

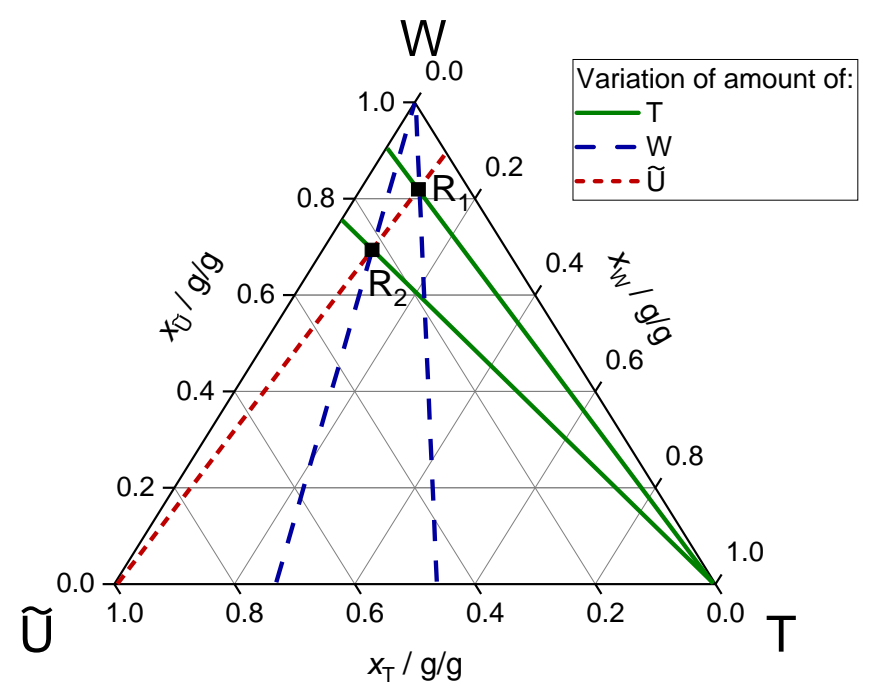

Figure 2: Schematic triangular diagram of a pseudo-ternary system $(T+W+\tilde{U})$. The black squares denote the compositions of two reference mixtures $R_{1}$ and $R_{2}$ and the colored lines denote the compositions of mixtures that are obtained by the variation of the amount of $T, W$, or $\tilde{U}$ starting from $R_{1}$ or $R_{2}$. The ratio of $T / W$ is the same in both reference mixtures, cf. Table 1

of which NMR spectra were taken. The reference mixtures were typically water-rich, cf. Table 1 . Starting from each reference mixture R, the amount of one of the three overall components $\mathrm{T}, \mathrm{W}$, or $\tilde{U}$ was varied whereas the ratio of the two others was held constant. This corresponds to an enrichment or a depletion of the chosen component and is indicated by the colored lines in Figure 2. The symbols indicating $R_{1}$ and $R_{2}$ lie on the red line since the ratio of $T / W$ is the same in both reference mixtures of each system, cf. Table 1. The predictions are compared with the results that were obtained with modified UNIFAC (Dortmund) using the full information on the true composition regarding the components $\mathrm{T}, \mathrm{W}$, and $\mathrm{U}_{1} \ldots \mathrm{U}_{N}$ and with results that were obtained in our previous work [19], in which an NMR analysis of each mixture was used. All results are shown as functions of the composition of the fully specified mixtures in the following.

\section{Results and Discussion}

In Figures 3-5, results for the activity coefficient of the target component ethanol in mixtures of system A (ethanol $(\mathrm{T})+$ water $(\mathrm{W})+2$-butanone $(\mathrm{U}))$ are shown. The predicted activity coefficients of $\mathrm{T}$ in the mixtures with varying compositions based on $\mathrm{R}_{1}$ were obtained with NEAT using only an NMR spectrum of $\mathrm{R}_{1}$; the same holds for $\mathrm{R}_{2}$.

In Figure 3 the influence of the variation of the amount of $\mathrm{T}$ on the activity coefficient of $\mathrm{T}$ is studied. The ratio $\tilde{U} / \mathrm{W}$ is held constant (cf. green lines in Figure 2). In all cases the predictions from NEAT are in excellent agreement with the results for the fully specified mixtures. This also 


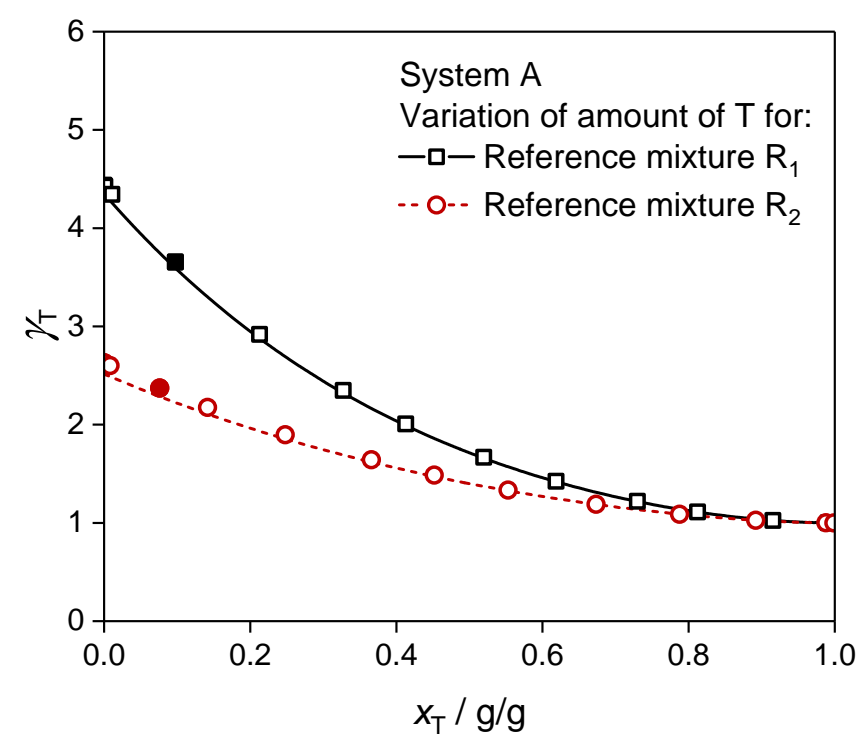

Figure 3: Activity coefficient $\gamma_{\mathrm{T}}$ of target component ethanol $(\mathrm{T})$ in ternary mixtures of $\mathrm{T}$ with water (W) and 2-butanone $(\mathrm{U})$. The amount of $\mathrm{T}$ is varied as shown in Figure 2 cf. green lines. Lines: results from modified UNIFAC (Dortmund) for the fully specified mixtures. Symbols: predictions from NEAT. The filled symbols indicate the reference mixtures, cf. Table 1 . No information on U was used in NEAT. $T=298 \mathrm{~K}$.

holds for the limiting activity coefficient of $\mathrm{T}$.

In Figure 4 , the influence of the variation of the amount of $\tilde{U}$ on the activity coefficient of $\mathrm{T}$ is studied. Since the mass ratio of $\mathrm{T} / \mathrm{W}$ is the same in both reference mixtures $\mathrm{R}_{1}$ and $\mathrm{R}_{2}$ (cf. red line in Figure 21, the results from modified UNIFAC (Dortmund) for the fully specified mixtures merge in one line. The predictions from NEAT are in very good agreement with the results for the fully specified mixtures. The predictions based on the NMR analysis of $R_{1}$ and $R_{2}$ are similarno significant influence of the choice of the reference mixture is found. Furthermore, no significant difference between the results of the present work and the results from our previous work 19 for which an NMR analysis of each studied mixture was used is found. Hence, the simplification of using only one NMR spectrum of a single mixture per system for the predictions with NEAT has basically no influence on the quality of the predictions.

In Figure 5 the influence of the variation of the amount of $\mathrm{W}$ on the activity coefficient of $\mathrm{T}$ is studied. The ratio $\tilde{\mathrm{U}} / \mathrm{T}$ is held constant (cf. blue lines in Figure 2). For water mass fractions approaching unity, the activity coefficient of $\mathrm{T}$ approaches the activity coefficient of $\mathrm{T}$ at infinite dilution in water since the influence of $\tilde{U}$ is vanishing. Hence, the curves merge for $x_{\mathrm{W}} \rightarrow 1 \mathrm{~g} / \mathrm{g}$. All trends are well represented by the predictions from NEAT and the agreement with the results for the fully specified mixtures is excellent. 


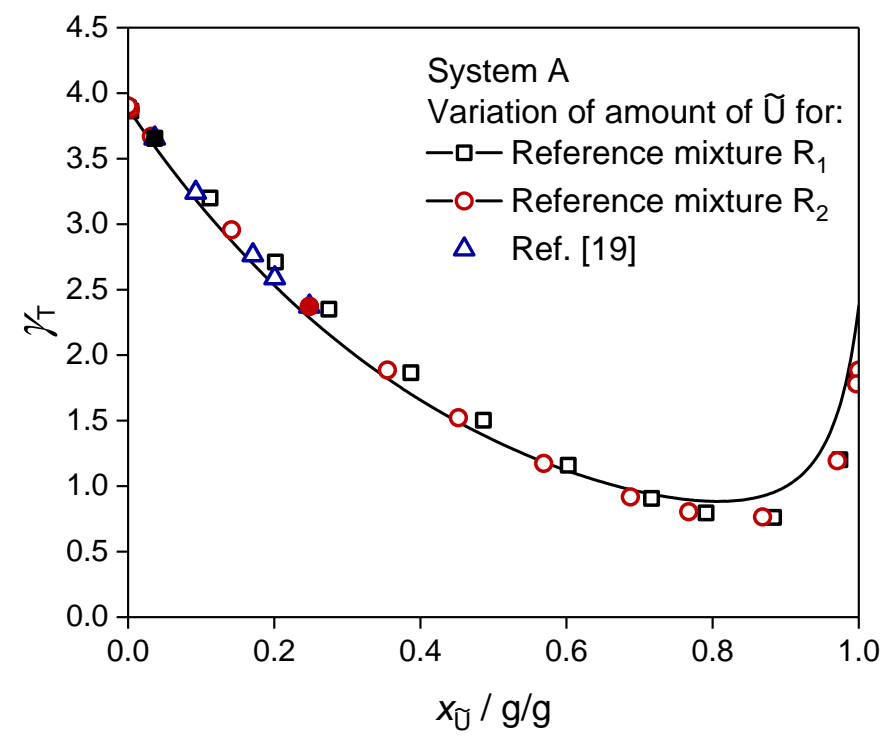

Figure 4: Activity coefficient $\gamma_{\mathrm{T}}$ of target component ethanol $(\mathrm{T})$ in ternary mixtures of $\mathrm{T}$ with water (W) and 2-butanone $(\mathrm{U})$. The amount of $\tilde{U}$ is varied as shown in Figure 2 cf. red line. Lines: results from modified UNIFAC (Dortmund) for the fully specified mixtures. Symbols: predictions from NEAT. The filled symbols indicate the reference mixtures, cf. Table 1 The blue triangles indicate results from our previous work 19. No information on $\mathrm{U}$ was used in NEAT. $T=298 \mathrm{~K}$.

Figures 6- 8 show results for the activity coefficient of the target component acetone in system $\mathrm{B}\left(\operatorname{acetone}(\mathrm{T})+\operatorname{water}(\mathrm{W})+\operatorname{xylose}\left(\mathrm{U}_{1}\right)+\operatorname{acetic}\right.$ acid $\left(\mathrm{U}_{2}\right)+$ methyl acetate $\left.\left(\mathrm{U}_{3}\right)\right)$. The presentation is similar to the one for system A.

In Figure 6, the influence of the variation of the amount of $\mathrm{T}$ on the activity coefficient of $\mathrm{T}$ is shown. The ratio $\tilde{\mathrm{U}} / \mathrm{W}$ is held constant (cf. green lines in Figure 2). The agreement of the predictions from NEAT with the results for the fully specified mixtures is excellent. Again, this also holds for the limiting activity coefficient of $\mathrm{T}$.

In Figure 7. the influence of the variation of the amount of $\tilde{\mathrm{U}}$ on the activity coefficient of $\mathrm{T}$ is studied. Since the mass ratio of $\mathrm{T} / \mathrm{W}$ is the same in both reference mixtures $\mathrm{R}_{1}$ and $\mathrm{R}_{2}$ (cf. red line in Figure 2, the results from modified UNIFAC (Dortmund) for the fully specified mixtures coincide again. No significant difference between the predictions from NEAT based on the NMR analysis of $R_{1}$ or $R_{2}$ and the predictions from our previous work, for which an NMR analysis of each mixture was used, is found. In all cases, the predictions from NEAT are very good.

In Figure 8 , the influence of the variation of the amount of $\mathrm{W}$ on the activity coefficient of $\mathrm{T}$ is studied. The ratio $\tilde{\mathrm{U}} / \mathrm{T}$ is held constant (cf. blue lines in Figure 2). For water mass fractions approaching unity, the activity coefficient of $\mathrm{T}$ approaches the activity coefficient of $\mathrm{T}$ at infinite dilution in water since the influence of $\tilde{U}$ is vanishing. Hence, the curves merge again for $x_{\mathrm{W}} \rightarrow$ $1 \mathrm{~g} / \mathrm{g}$. The agreement of all predictions from NEAT with the results for the fully specified mixtures is very good. 


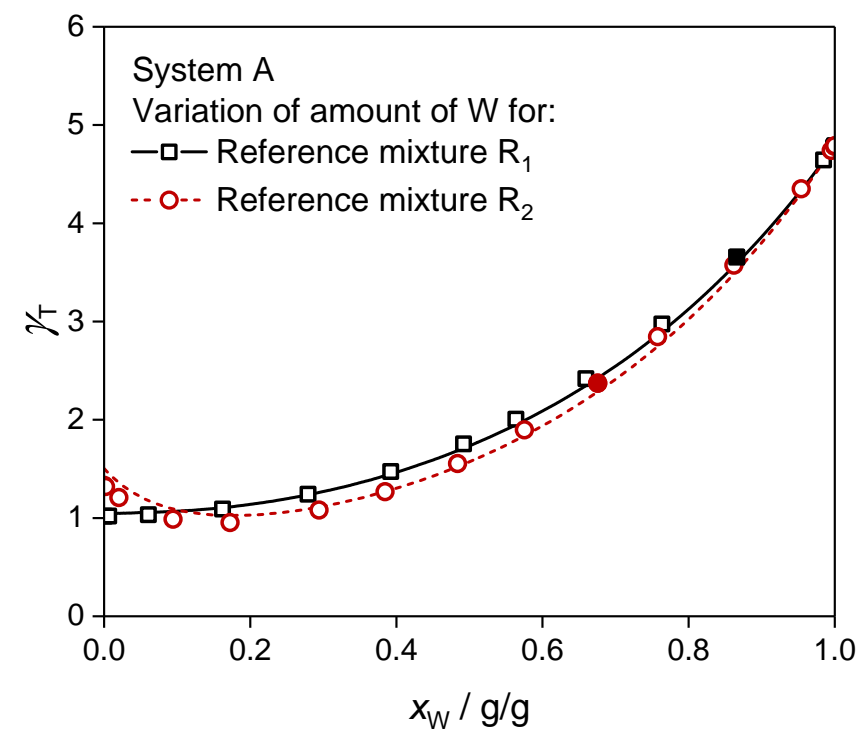

Figure 5: Activity coefficient $\gamma_{\mathrm{T}}$ of target component ethanol $(\mathrm{T})$ in ternary mixtures of $\mathrm{T}$ with water $(\mathrm{W})$ and 2butanone (U). The amount of $\mathrm{W}$ is varied as shown in Figure 2 cf. blue lines. Lines: results from modified UNIFAC (Dortmund) for the fully specified mixtures. Symbols: predictions from NEAT. The filled symbols indicate the reference mixtures, cf. Table 1 No information on $\mathrm{U}$ was used in NEAT. $T=298 \mathrm{~K}$.

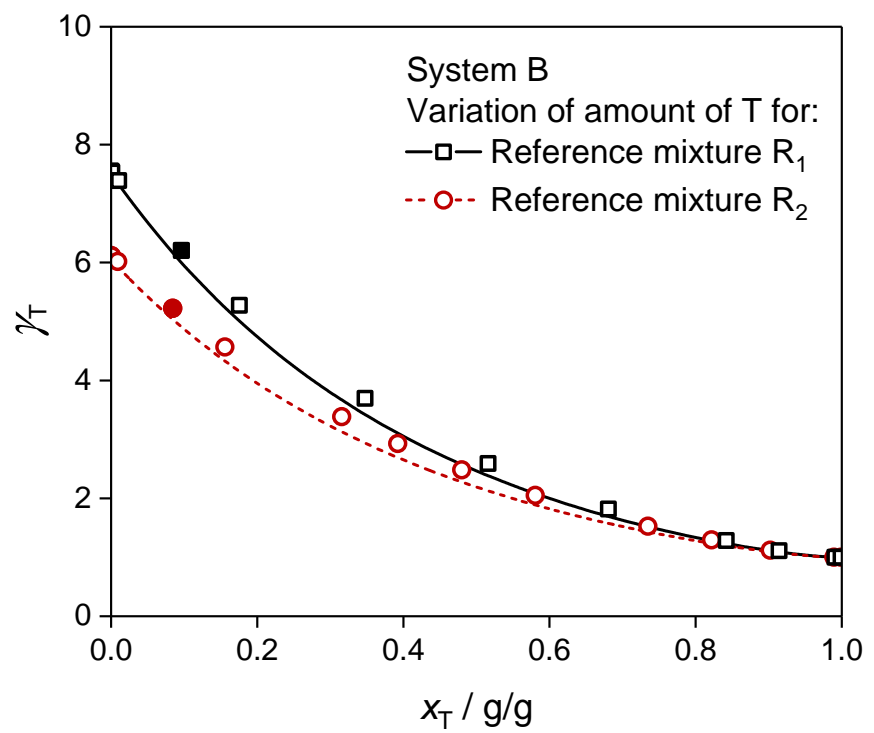

Figure 6: Activity coefficient $\gamma_{\mathrm{T}}$ of target component acetone (T) in five-component mixtures of $\mathrm{T}$ with water $(\mathrm{W})$, xylose $\left(\mathrm{U}_{1}\right)$, acetic acid $\left(\mathrm{U}_{2}\right)$, and methyl acetate $\left(\mathrm{U}_{3}\right)$. The amount of $\mathrm{T}$ is varied as shown in Figure 2 cf. green lines. Lines: results from modified UNIFAC (Dortmund) for the fully specified mixtures. Symbols: predictions from NEAT. The filled symbols indicate the reference mixtures, cf. Table 1 No information on U was used in NEAT. $T=298 \mathrm{~K}$ 


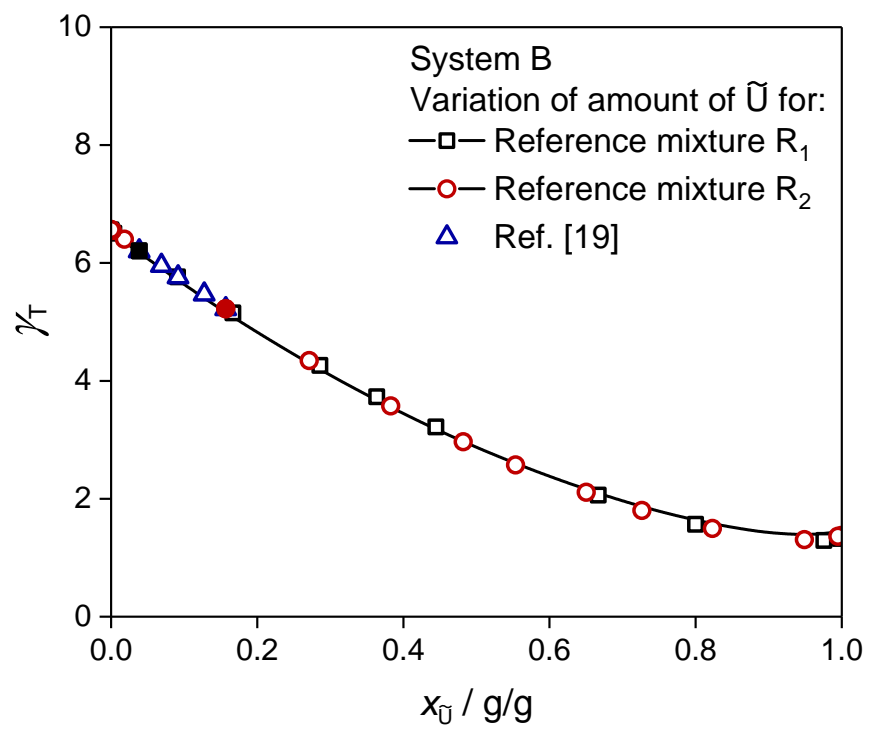

Figure 7: Activity coefficient $\gamma_{\mathrm{T}}$ of target component acetone $(\mathrm{T})$ in five-component mixtures of $\mathrm{T}$ with water $(\mathrm{W})$, xylose $\left(\mathrm{U}_{1}\right)$, acetic acid $\left(\mathrm{U}_{2}\right)$, and methyl acetate $\left(\mathrm{U}_{3}\right)$. The amount of $\tilde{\mathrm{U}}$ is varied as shown in Figure 2 cf. red line. Lines: results from modified UNIFAC (Dortmund) for the fully specified mixtures. Symbols: predictions from NEAT. The filled symbols indicate indicate the reference mixtures, cf. Table 1 The blue triangles indicate results from our previous work [19]. No information on U was used in NEAT. $T=298 \mathrm{~K}$.

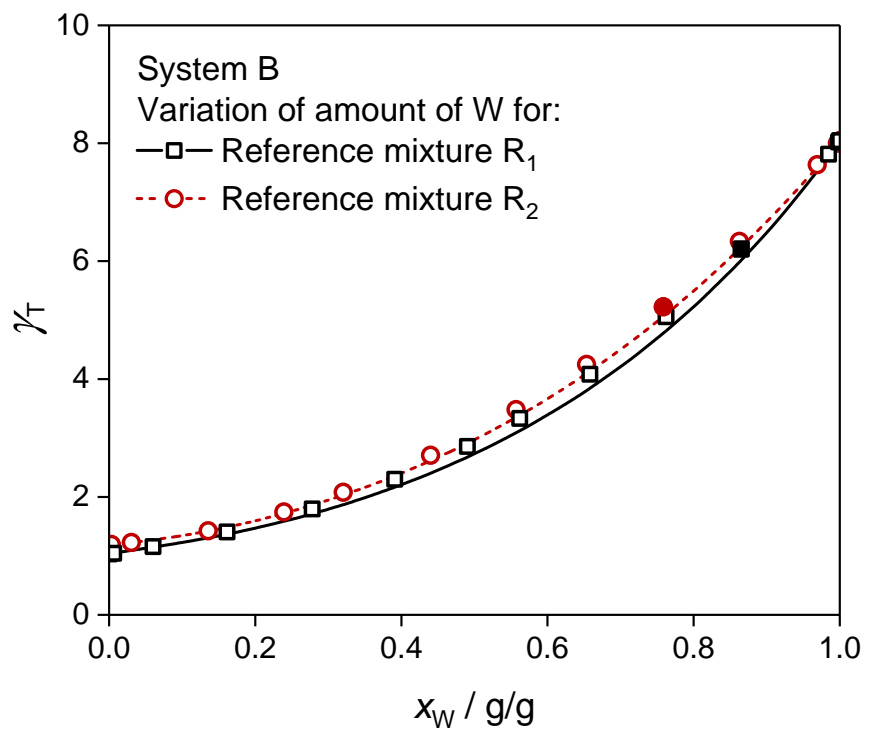

Figure 8: Activity coefficient $\gamma_{\mathrm{T}}$ of target component acetone (T) in five-component mixtures of $\mathrm{T}$ with water (W), xylose $\left(\mathrm{U}_{1}\right)$, acetic acid $\left(\mathrm{U}_{2}\right)$, and methyl acetate $\left(\mathrm{U}_{3}\right)$. The amount of $\mathrm{W}$ is varied as shown in Figure 2 cf. blue lines. Lines: results from modified UNIFAC (Dortmund) for the fully specified mixtures, cf. Table 1 . Symbols: predictions from NEAT. The filled symbols indicate the reference mixtures. No information on U was used in NEAT. $T=298 \mathrm{~K}$. 
In Figures 9-11, results for the activity coefficient of the target component 1,4-butanediol in system $\mathrm{C}\left(1,4\right.$-butanediol $(\mathrm{T})+$ water $(\mathrm{W})+$ cyclohexanone $\left(\mathrm{U}_{1}\right)+$ acetonitrile $\left(\mathrm{U}_{2}\right)+$ methyl acetate $\left.\left(\mathrm{U}_{3}\right)\right)$ are shown. The presentation is similar to the one for the previous examples.

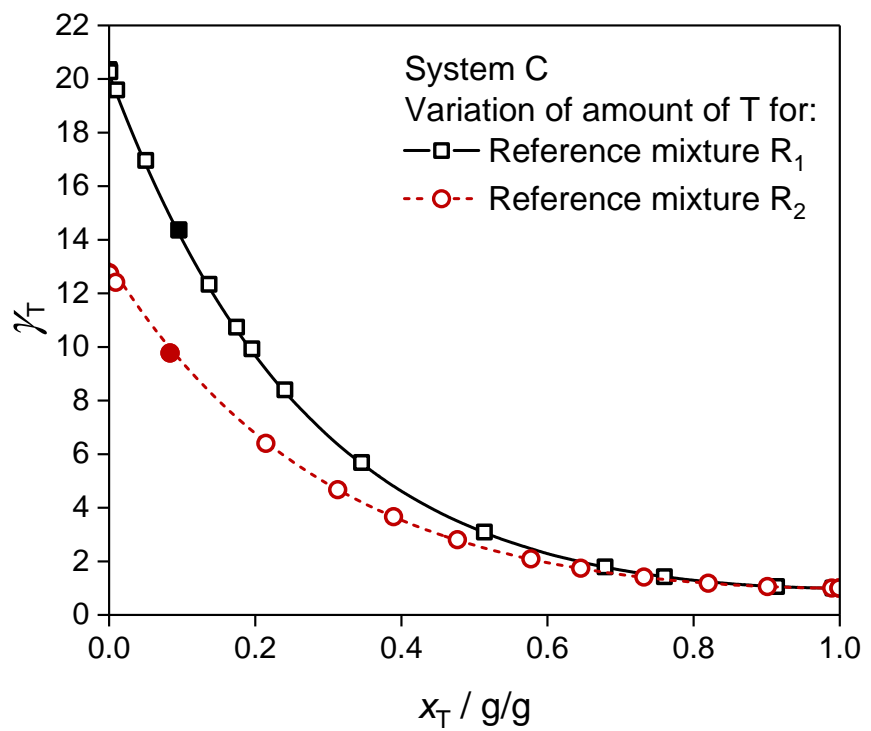

Figure 9: Activity coefficient $\gamma_{\mathrm{T}}$ of target component 1,4-butanediol (T) in five-component mixtures of $\mathrm{T}$ with water $(\mathrm{W})$, cyclohexanone $\left(\mathrm{U}_{1}\right)$, acetonitrile $\left(\mathrm{U}_{2}\right)$, and methyl acetate $\left(\mathrm{U}_{3}\right)$. The amount of $\mathrm{T}$ is varied as shown in Figure 2, cf. green lines. Lines: results from modified UNIFAC (Dortmund) for the fully specified mixtures. Symbols: predictions from NEAT. The filled symbols indicate the reference mixtures, cf. Table 1 No information on $\mathrm{U}$ was used in NEAT. $T=298 \mathrm{~K}$.

In Figure 9, the influence of the variation of the amount of $\mathrm{T}$ on the activity coefficient of $\mathrm{T}$ is shown. The agreement of the predictions from NEAT for the poorly specified mixtures with the results from modified UNIFAC (Dortmund) for the fully specified mixtures is almost perfect, including the predictions for the limiting activity coefficient of $\mathrm{T}$.

In Figure 10, the influence of the variation of the amount of $\tilde{U}$ on the activity coefficient of $\mathrm{T}$ is shown. Since the mass ratio of $\mathrm{T} / \mathrm{W}$ is the same in both reference mixtures $\mathrm{R}_{1}$ and $\mathrm{R}_{2}$ (cf. red line in Figure 2), the results from modified UNIFAC (Dortmund) for the fully specified mixtures merge again in one line. Up to a mass fraction of $x_{\tilde{\mathrm{U}}}=0.5 \mathrm{~g} / \mathrm{g}$, the agreement of the predictions with NEAT with the results for the fully specified mixtures is very good. At higher mass fractions of $\tilde{U}$, the deviations increase. However, the trends are represented well throughout. Again, no significant influence of the choice of the reference mixture and similar results as in our previous work [19, in which an NMR analysis of each mixture was used, are found.

In Figure 11, the influence of the variation of the amount of $\mathrm{W}$ on the activity coefficient of $\mathrm{T}$ is shown. The ratio $\tilde{\mathrm{U}} / \mathrm{T}$ is held constant (cf. blue lines in Figure 2). For water mass fractions approaching unity, the activity coefficient of $\mathrm{T}$ approaches the activity coefficient of $\mathrm{T}$ at infinite dilution in water since the influence of $\tilde{U}$ is vanishing. Hence, the curves merge again for $x_{\mathrm{W}} \rightarrow$ 


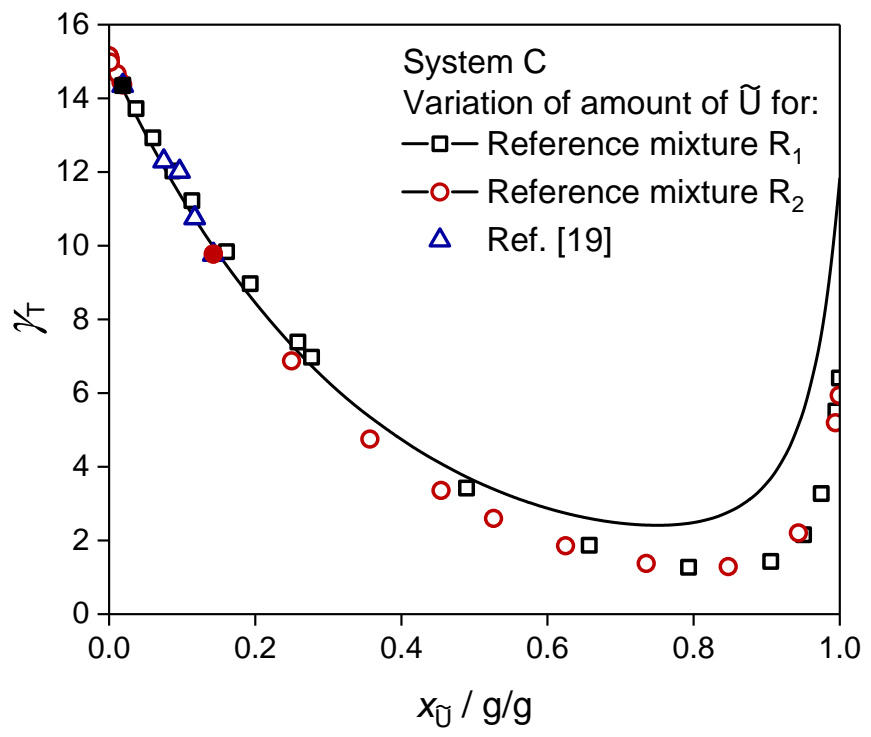

Figure 10: Activity coefficient $\gamma_{\mathrm{T}}$ of target component 1,4-butanediol $(\mathrm{T})$ in five-component mixtures of $\mathrm{T}$ with water $(W)$, cyclohexanone $\left(\mathrm{U}_{1}\right)$, acetonitrile $\left(\mathrm{U}_{2}\right)$, and methyl acetate $\left(\mathrm{U}_{3}\right)$. The amount of $\tilde{U}$ is varied as shown in Figure 2 cf. red line. Lines: results from modified UNIFAC (Dortmund) for the fully specified mixtures. Symbols: predictions from NEAT. The filled square and circle indicate the reference mixtures, cf. Table 1 The blue triangles indicate results from our previous work [19]. No information on U was used in NEAT. T $=298 \mathrm{~K}$.

$1 \mathrm{~g} / \mathrm{g}$. The agreement of all predictions from NEAT with the results for the fully specified mixtures is almost perfect. 


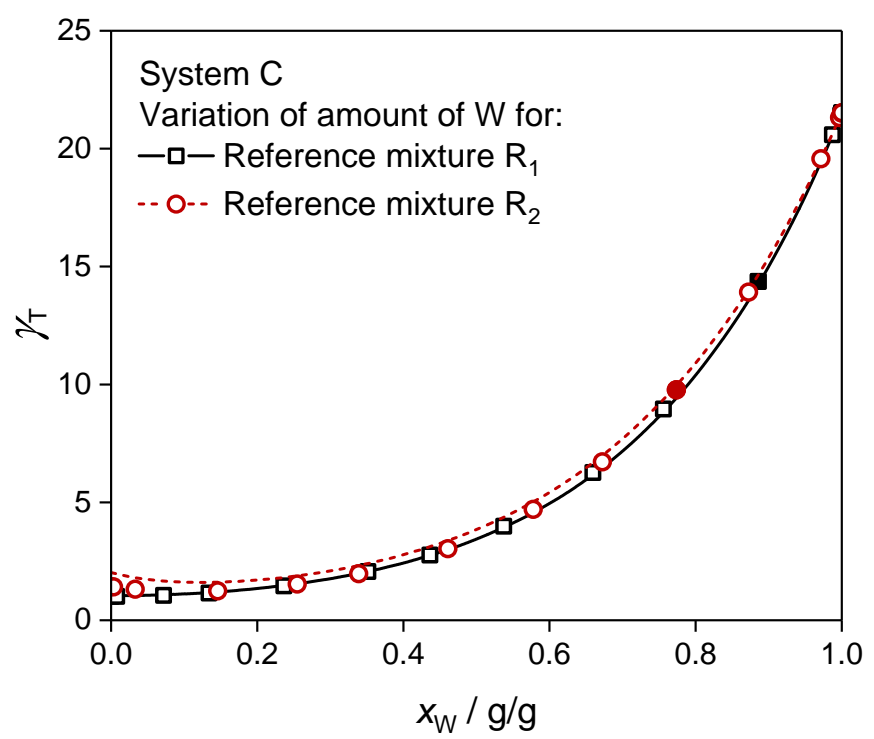

Figure 11: Activity coefficient $\gamma_{\mathrm{T}}$ of target component 1,4-butanediol $(\mathrm{T})$ in five-component mixtures of $\mathrm{T}$ with water $(\mathrm{W})$, cyclohexanone $\left(\mathrm{U}_{1}\right)$, acetonitrile $\left(\mathrm{U}_{2}\right)$, and methyl acetate $\left(\mathrm{U}_{3}\right)$. The amount of $\mathrm{W}$ is varied as shown in Figure 2 cf. blue lines. Lines: results from modified UNIFAC (Dortmund) for the fully specified mixtures. Symbols: predictions from NEAT. The filled symbols indicate the reference mixtures, cf. Table 1 No information on U was used in NEAT. $T=298 \mathrm{~K}$. 


\section{Conclusions}

In a previous work of our group, the NEAT method to predict activity coefficients of target components in poorly specified mixtures was introduced. It is based on a quantitative NMR analysis to identify the chemical groups of the unknown components in the mixture and the thermodynamic group contribution method modified UNIFAC (Dortmund). In this work, we show that, based on one NMR analysis of a single mixture, the activity coefficient of the target component in all mixtures of the same system can be predicted with NEAT as long as the ratios between the amounts of the unknown components in the mixtures remain unchanged. This is the case in many practical problems, such as the selective separation of the target component from a liquid mixture or the removal of solvent to increase the concentration of a diluted solution. The quality of the predictions that are obtained with NEAT is similar to the one that is achieved when modified UNIFAC (Dortmund) is applied to fully specified mixtures. NEAT is particularly interesting for the model-based design of processes with poorly specified mixtures. The present work shows how NEAT can be applied in a particularly simple manner to an important class of problems.

\section{Funding Sources} work Program for Research and Innovation Horizon 2020 (2014-2020) under grant agreement no. 637077.

\section{Declarations of interest}

none 


\section{Nomenclature}

Symbols

$M \quad$ molar mass / g/mol

$N \quad$ number of unknown components

$\mathrm{R}$ reference mixture

$\mathrm{T}$ target component

$T$ temperature / $\mathrm{K}$

U unknown component

$\tilde{U}$ mean unknown component

W water

$x \quad$ mass fraction / g/g

Greek letters

$\gamma$ activity coefficient

Abbreviations

COSMO-RS Conductor like Screening Model for Real Solvents

NEAT NMR Spectroscopy for the Estimation of Activity Coefficients of Target Components in Poorly Specified Mixtures

NMR Nuclear Magnetic Resonance

NRTL Non-Random Two-Liquid Model

UNIFAC Universal Quasichemical Functional Group Activity Coefficient Model

UNIQUAC Universal Quasichemical Model

\section{References}

225 [1] G. L. Alexander, A. L. Creagh, J. M. Prausnitz, Phase Equilibria for High-Boiling Fossil-Fuel Distillates. 1. Characterization, Ind. Eng. Chem. Fundam. 24 (1985) 301-310.

[2] M. A. Fahim, A. S. Elkilani, Prediction of Solubility of Hydrogen in Petroleum Cuts using Modified UNIFAC, Can. J. Chem. Eng. 70 (2) (1992) 335-340.

[3] E. Darwish, T. A. Al-Sahhaf, M. A. Fahim, Prediction of the Surface Tension of Petroleum Cuts using a Modified UNIFAC Group Contribution Method, Fluid Phase Equilib. 105 (2) (1995) 229-239. 
[4] B. Carreón-Calderón, V. Uribe-Vargas, E. Ramírez-Jaramillo, M. Ramírez-de Santiago, Thermodynamic Characterization of Undefined Petroleum Fractions using Group Contribution Methods, Ind. Eng. Chem. Res. 51 (43) (2012) 14188-14198.

[12] G. Maurer, J. M. Prausnitz, On the Derivation and Extension of the UNIQUAC Equation, Fluid Phase Equilib. 2 (2) (1978) 91-99.

[13] A. Fredenslund, R. L. Jones, J. M. Prausnitz, Group-contribution Estimation of Activity Coefficients in Nonideal Liquid Mixtures, AIChE J. 21 (6) (1975) 1086-1099.

[14] A. Fredenslund, J. Gmehling, P. Rasmussen, Vapor-Liquid Equilibria using UNIFAC, a GroupContribution Method, 1st Edition, Elsevier, Amsterdam, The Netherlands, 1977.

[15] D. Constantinescu, J. Gmehling, Further Development of Modified UNIFAC (Dortmund): Revision and Extension 6, J. Chem. Eng. Data 61 (8) (2016) 2738-2748.

[16] A. Klamt, Conductor-Like Screening Model for Real Solvents: A New Approach to the Quantitative Calculation of Solvation Phenomena, J. Phys. Chem. 99 (7) (1995) 2224-2235. 
[17] A. Klamt, V. Jonas, T. Bürger, J. C. W. Lohrenz, Refinement and Parametrization of COSMO-RS, J. Phys. Chem. A 102 (26) (1998) 5074-5085.

[18] F. Jirasek, J. Burger, H. Hasse, Method for Estimating Activity Coefficients of Target Components in Poorly Specified Mixtures, Ind. Eng. Chem. Res. 57 (21) (2018) 7310-7313.

[19] F. Jirasek, J. Burger, H. Hasse, NEAT - NMR Spectroscopy for the Estimation of Activity Coefficients of Target Components in Poorly Specified Mixtures, Ind. Eng. Chem. Res. (accepted). doi:10.1021/acs.iecr.9b01269.

\section{Appendix A. Assignment of Chemical Groups to NMR Chemical Shift Regions}

Table A.1 shows the assignment of chemical groups from the modified UNIFAC (Dortmund) table to ${ }^{13} \mathrm{C}$ NMR chemical shift regions that was used in the present work. The assignment is the same as the one that was used in a previous work of our group Ref. [19] to predict the activity coefficients of the target components in aqueous poorly specified mixtures. In addition to the information on the unknown components, the mass fraction of water in the poorly specified mixture is determined from the mass balance.

Table A.1: Assignment of chemical groups to chemical shift regions of ${ }^{13} \mathrm{C}$ NMR spectra used in this work. All groups are groups from the modified UNIFAC (Dortmund) table. The mass fraction of water (last row) in all reference mixtures $\mathrm{R}$ was calculated from the mass balance.

\begin{tabular}{|c|c|c|}
\hline${ }^{13} \mathrm{C}$ NMR chemical shift region & Group assignment & UNIFAC label \\
\hline $0-60 \mathrm{ppm}$ & alkyl, carbonyl & '대2', '르2CO' \\
\hline $60-90 \mathrm{ppm}$ & alcohol & ' $\underline{\mathrm{CH}} 2$ ' + 'OH$(\mathrm{P})$ ' \\
\hline $90-150 \mathrm{ppm}$ & alkenyl & ' $\underline{\mathrm{CH}}=\underline{\mathrm{CH}} '$ \\
\hline $150-180 \mathrm{ppm}$ & carboxyl & 'ㅇOH' \\
\hline$>180 \mathrm{ppm}$ & carbonyl & 'CH2 $\underline{\mathrm{CO}}$ ' \\
\hline n.a. & water & ' $\mathrm{H} 2 \mathrm{O} '$ \\
\hline
\end{tabular}

\section{Appendix B. Results for the Fully Specified Mixtures}

In Figure B.1. results for the activity coefficient $\gamma_{\mathrm{T}}$ of the target component ethanol (T) in mixtures of system A calculated with modified UNIFAC (Dortmund) for the fully specified mixtures (left) and the absolute deviations between the results for the fully specified mixtures and the predictions with NEAT, cf. Figure 1. (right) are shown. Very similar surfaces and respectively small deviations are found. The largest deviations occur for high concentrations of the unknown component ( $\mathrm{U}=2$-butanone) for which no information was used in NEAT, cf. edge labeled with $\tilde{\mathrm{U}}$. 

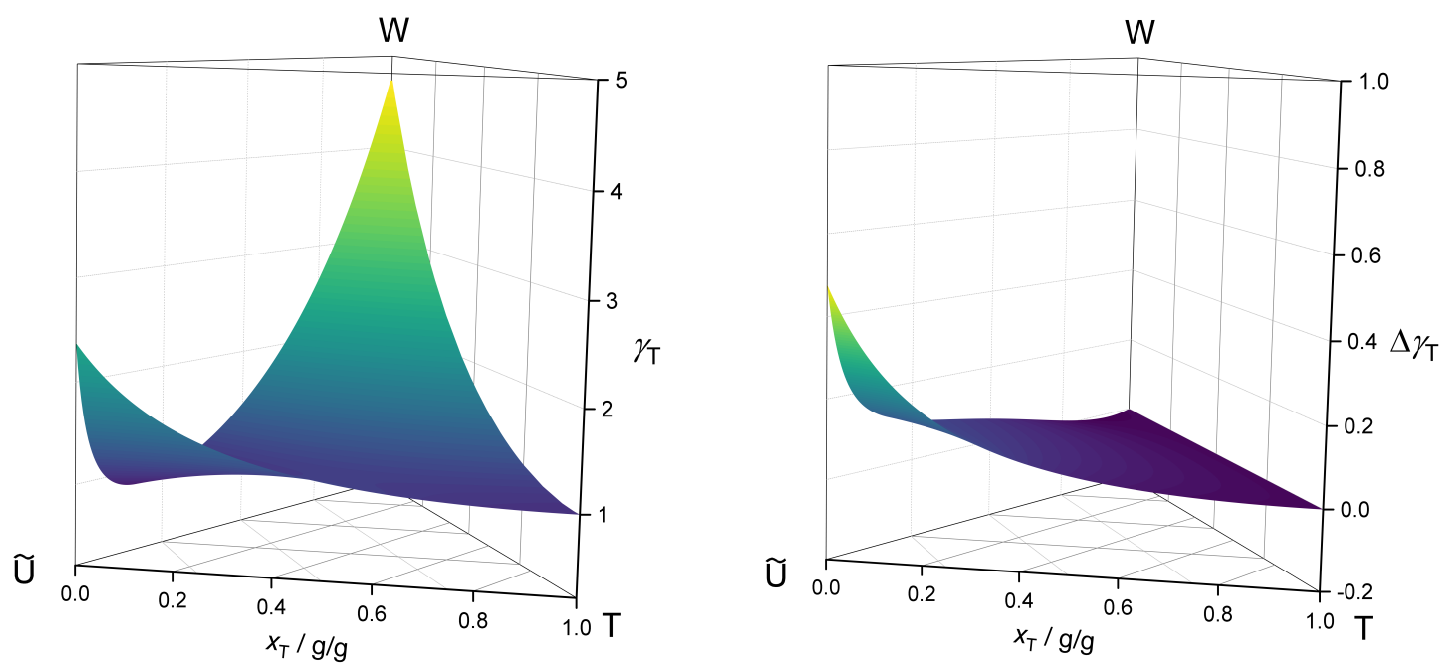

Figure B.1: Left: activity coefficient $\gamma_{\mathrm{T}}$ of target component ethanol $(\mathrm{T})$ in mixtures of system A calculated with modified UNIFAC (Dortmund) for the fully specified mixtures. Right: absolute deviations $\Delta \gamma_{\mathrm{T}}$ between the predictions with NEAT, cf. Figure 1 in the main text, and the results for the fully specified mixtures. $\Delta \gamma_{\mathrm{T}}=$ $\left(\gamma_{\mathrm{T}}\right)^{\mathrm{UNIFAC}}-\left(\gamma_{\mathrm{T}}\right)^{\mathrm{NEAT}} . T=298 \mathrm{~K}$

\section{Appendix C. Estimated Composition of the Reference Mixtures}

In Tables C.1- C.3. the estimated composition of the poorly specified reference mixtures and the estimated stoichiometry of the mean unknown component $\tilde{U}$ as obtained with NEAT for the studied systems are summarized.

Table C.1: Estimated composition in the component space (target component $\mathrm{T}+$ water $\mathrm{W}+$ mean unknown component $\tilde{U}$ ) for the reference mixtures of system A (ethanol $(\mathrm{T})+$ water $(\mathrm{W})+2$-butanone $(\mathrm{U})$ ) and stoichiometry of $\tilde{\mathrm{U}}$ for $M_{\tilde{\mathrm{U}}}=150 \mathrm{~g} / \mathrm{mol}$ obtained with NEAT. The labels are the identifiers for the subgroup from modified UNIFAC (Dortmund).

\begin{tabular}{c|c|c|c|c|c|c|c}
\hline \hline & \multicolumn{2}{|c|}{$x_{i} / \mathrm{g} / \mathrm{g}$} & \multicolumn{5}{c}{ Stoichiometry of U } \\
\hline Mixture & $x_{\mathrm{T}}$ & $x_{\tilde{\mathrm{U}}}$ & 'CH2' & 'OH(P)' & 'HC=CH' & 'COOH' & 'CH2CO' \\
\hline R1 & 0.098 & 0.029 & 4.743 & 0.000 & 0.000 & 0.000 & 1.986 \\
R2 & 0.076 & 0.229 & 4.442 & 0.000 & 0.000 & 0.000 & 2.086 \\
\hline \hline
\end{tabular}

Table C.2: Estimated composition in the component space (target component $\mathrm{T}+$ water $\mathrm{W}+$ mean unknown component $\tilde{U})$ for the reference mixtures of system B (acetone $(T)+$ water $(W)+$ xylose $\left(U_{1}\right)+$ acetic acid $\left(U_{2}\right)$ + methyl acetate $\left.\left(\mathrm{U}_{3}\right)\right)$ and stoichiometry of $\tilde{\mathrm{U}}$ for $M_{\tilde{\mathrm{U}}}=150 \mathrm{~g} / \mathrm{mol}$ obtained with NEAT. The labels are the identifiers for the subgroup from modified UNIFAC (Dortmund).

\begin{tabular}{c|c|c|c|c|c|c|c}
\hline \hline & \multicolumn{2}{|c|}{$x_{i} / \mathrm{g} / \mathrm{g}$} & \multicolumn{5}{c}{ Stoichiometry of $\tilde{\mathrm{U}}$} \\
\hline Mixture & $x_{\mathrm{T}}$ & $x_{\tilde{\mathrm{U}}}$ & 'CH2' & 'OH(P)' & 'HC=CH' & 'COOH' & 'CH2CO' \\
\hline R1 & 0.096 & 0.031 & 3.640 & 1.266 & 0.237 & 1.582 & 0.000 \\
R2 & 0.084 & 0.133 & 3.772 & 1.378 & 0.164 & 1.541 & 0.000 \\
\hline \hline
\end{tabular}


Table C.3: Estimated composition in the component space (target component $\mathrm{T}+$ water $\mathrm{W}+$ mean unknown component $\tilde{U})$ for the reference mixtures of system $\mathrm{C}\left(1,4\right.$-butanediol $(\mathrm{T})+$ water $(\mathrm{W})+$ cyclohexanone $\left(\mathrm{U}_{1}\right)+$ acetonitrile $\left(\mathrm{U}_{2}\right)+$ methyl acetate $\left.\left(\mathrm{U}_{3}\right)\right)$ and stoichiometry of $\tilde{\mathrm{U}}$ for $M_{\tilde{\mathrm{U}}}=150 \mathrm{~g} / \mathrm{mol}$ obtained with NEAT. The labels are the identifiers for the subgroup from modified UNIFAC (Dortmund).

\begin{tabular}{c|c|c|c|c|c|c|c}
\hline \hline & \multicolumn{2}{|c|}{$x_{i} / \mathrm{g} / \mathrm{g}$} & \multicolumn{5}{c}{ Stoichiometry of $\tilde{\mathrm{U}}$} \\
\hline Mixture & $x_{\mathrm{T}}$ & $x_{\tilde{\mathrm{U}}}$ & ${ }^{\prime} \mathrm{CH} 2$ & ${ }^{\prime} \mathrm{OH}(\mathrm{P})^{\prime}$ & ${ }^{\prime} \mathrm{HC}=\mathrm{CH}$ & ${ }^{\prime} \mathrm{COOH}$ & ${ }^{\prime} \mathrm{CH} 2 \mathrm{CO}{ }^{\prime}$ \\
\hline $\mathrm{R} 1$ & 0.096 & 0.013 & 5.793 & 0.000 & 0.653 & 0.666 & 0.518 \\
$\mathrm{R} 2$ & 0.083 & 0.121 & 5.414 & 0.000 & 0.675 & 0.775 & 0.514 \\
\hline \hline
\end{tabular}

